\title{
Distributed Electricity Markets and Distribution Locational Marginal Prices: A Review
}

\author{
Calum Edmunds \\ University of Strathclyde, Scotland \\ calum.edmunds@strath.ac.uk
}

\author{
Dr Stuart Galloway \\ University of Strathclyde, Scotland \\ stuart.galloway@strath.ac.uk
}

\begin{abstract}
As part of the transition from distribution network operator to distribution system operator (DSO), decentralised pricing of energy is an area that needs to be considered. This paper considers different roles for the DSO in facilitating distributed markets including the decentralised and centralised approaches. The latest work in distributed markets has been reviewed including two major pilot projects involving DSO type operation of distribution markets. The use of distribution locational marginal prices (DLMPs) in distributed markets has been studied as a promising means of directing investment and managing constraints in distribution systems. Some simple case studies involving DLMPs in an example distribution network were modelled using Matpower and it was seen that marginal prices could result in reduced losses and congestion if DLMPs are passed on to distributed energy resources. A case was considered of a bus with losses resulting in DLMPs $6 \%$ above the grid import cost and it was seen if the DLMP market was used a generator installed at this bus could reduce the cost of losses by up to $14 \%$.
\end{abstract}

Index Terms-Locational Marginal Pricing, Distributed Generation, Electricity Markets

\section{INTRODUCTION}

I $\mathrm{N}$ the UK, distributed generation (DG) has increased by $54 \%$ between 2011 and 2014 up to $19.1 \mathrm{GW}$ in 2014 [1]. A major driver for this growth is the decarbonisation of electricity through the introduction of renewable technologies such as wind and solar embedded in distribution networks. It is anticipated that DG could contribute between 31 and $57 \mathrm{GW}$ of generation capacity in the UK by 2036 which could equate to up to $40 \%$ of installed capacity in 2036 [2]. The scale of this growth poses a significant challenge to the network operators. Without credible alternatives, significant grid reinforcement would be required to allow a fraction of this DG to reach areas of highest demand (often far from distributed resources) and maintain grid stability with increasing penetration of intermittent generation. However this creates a chance for new approaches to be considered. This paper will look at the opportunities brought about through distributed electricity markets. An illustration of the possible future interaction of distributed markets with the existing system is shown in Fig. 1.

The main benefits that can be gained from distributed markets are increasing the amount of DG that can be connected at distribution level and incentivising flexible demand. At present, the amount of generation that can be connected to distribution networks is based on the difference between maximum loading and the line capacity. There is limited active monitoring of distribution systems and the operational limits are based on worst case scenarios (e.g. maximum generation and minimum demand) which will occur relatively infrequently.

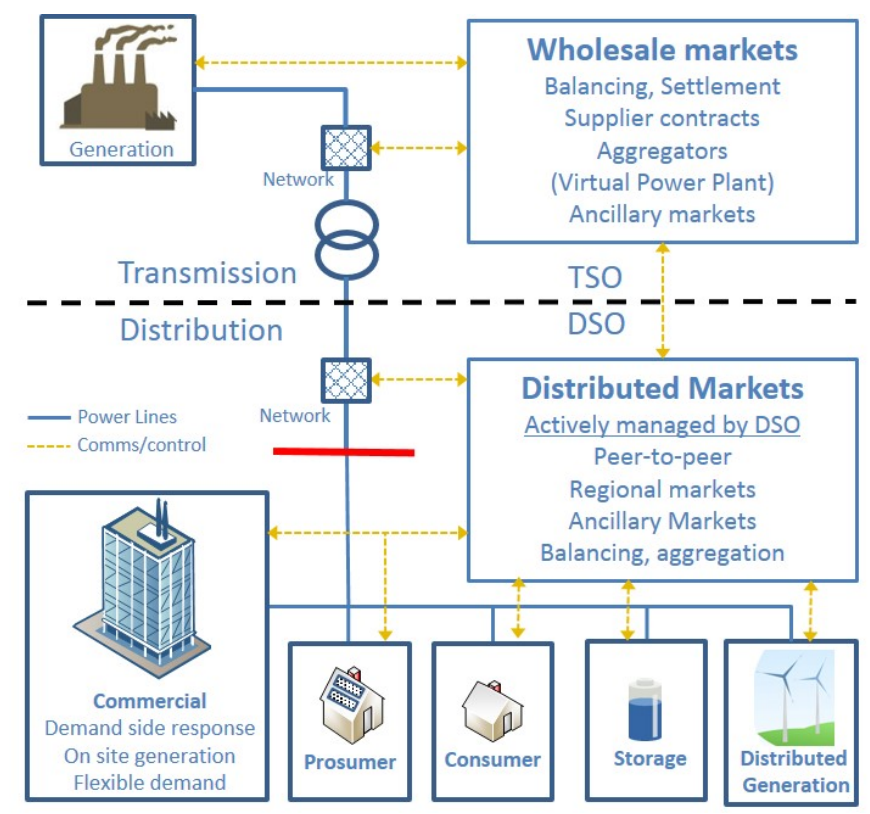

Fig. 1: Interaction of wholesale and distributed markets

With the roll out of smart meter technology it may become possible to actively manage distribution systems by communicating demand and generation from distributed energy resources (DERs) down to the level of individual households [3]. With increasing numbers of smart devices it is also considered possible to provide demand response from a large number of small loads which can be aggregated and coordinated by a DSO [4]. If smart meter data is available with sufficient granularity then there is potential to provide close to real-time supply/demand data which can be used to inform price signals in distributed markets. Using these price signals and a more actively managed distribution network, more DERs can be connected and line capacities can be maximised thus reducing the costs of reinforcement and furthermore, providing energy pricing that is more reflective of the costs at the distributed sources. For example at times of minimum demand the output from generators can be curtailed (as in active network 
management [5]) or price signals from distributed markets can encourage increased demand at times of peak generation.

An established method of providing effective price signals that vary spatially and temporally is locational marginal prices (LMPs) which are calculated at network locations including the effect of constraints and losses [6]. LMPs have the potential to play a central role in distributed markets by providing a tool for the DSO to coordinate local trades, reduce system constraints and direct network investment. This paper presents a review of the current state of the art in distributed electricity markets and the application of variable pricing to distribution networks. The work is based on the UK electricity system however references are made to research and commercial projects in Europe and the US. The structure of the paper is as follows: future distribution system operation is described in Section II, a review of the latest projects is presented in Section III, a study of DLMPs is summarised in IV and conclusions are drawn in Section V.

\section{Future Distributed System Operation}

There are few actively managed distribution systems known to the author at this time (although several pilot projects are in operation - see Section III). Work has been done in proposing possible distributed market structures and roles for the transmission system operator (TSO) and DSO in coordinating these markets. An in depth report on the structures of actively managed distribution systems has been carried out by Martini et al. [7] from the perspective of the Californian distribution networks. In this work different options for the structure of the actively managed distribution system are presented, these include;

- Total TSO - The TSO controls and operates the electricity system down to distribution level and optimises dispatch of DERs.

- Minimal DSO - The TSO does not optimise dispatch beyond the grid supply points (GSPs) or transmission distribution interface.

- Market DSO - In this model the DERs are aggregated to a minimum size (e.g $10 \mathrm{MW}$ ) which means the DSO is responsible for coordination of the DER aggregators within each distribution network and responding to dispatch instructions from the TSO.

It is concluded that the Total TSO is not the most efficient mode of operation and that the complexity of optimising dispatch at such a high level of granularity by a central TSO would make this option undesirable. An alternative is for a DSO to coordinate the DERs in response to TSO dispatch instructions which requires continuous real-time communication between the DSO and the TSO. The DSO can also become the balancing authority, balancing supply/demand in the local distribution area and importing/exporting to the transmission system when needed.
The market DSO model has potential for the integration of distributed markets in a similar manner to how the wholesale markets are coordinated by the TSO in centralised markets such as Nord Pool [8] or in the USA.

\section{STATE OF THE ART PROJECTS}

Distributed markets have seen significant development in recent years with a number of research, pilot and commercial projects [9]. A summary of some of the recent commercial and pilot scale projects taking place worldwide involving distributed markets is shown in Table I. The remainder of this section provides some more detail on two of the pilot projects presented in this table: Ecogrid and TDI 2.

TABLE I: Existing distributed markets projects

\begin{tabular}{lll} 
Project & Location & Scale \\
\hline Vandebron [10] & NL & Commercial
\end{tabular}

Peer-to-peer (P2P) AirBnB style site to connect renewable energy generators directly to demand. Cuts out supplier 'middle-man'. In 2015 there were 16 farms with online profiles supplying 20,000 customers.

Sonnen Community [11] Germany Commercial

Using SonnenBattery, community sharing power from PV systems, cuts out energy provider. 20 Euros/month membership fee (and upwards) but no standing charge.

TransActive grid [12] New York, USA Start-up

P2P trading of electricity using 'Ethereum' blockchain software. Five homes on one side of street with solar panels sell excess energy to homes on other side of street.

Open Utility [13] Cornwall, UK Pilot

Trial collaboration between Good Energy (100\% renewable supplier) and Open Utility. P2P energy marketplace (called Piclo) matching renewable generators to demands. Selectricity product now launched by Good Energy for businesses and generators based on Piclo product.

Power Ledger [14] Perth, Australia Pilot

$\mathrm{P} 2 \mathrm{P}$ trial using blockchain to authenticate decentralised transactions. 8 week trial with 10 houses and 20 people in Perth. To be trialed in New Zealand with energy company Vector on 500 sites in Auckland.

PowerMatching City Groningen, NL Pilot

[15]

Street with demand side response (DSR) matching power consumption to local generation from solar panels. Some homes have CHP and others have heat pumps with buffer storage tanks for demand shifting.

Ecogrid [3] Bornholm, Denmark Pilot

Danish Island with DSR using smart meters for 1900 domestic customers + industry. Includes 444 automated households and 654 semi-automated households.

Cornwall Local Energy Cornwall, UK Pilot Market [16]

Trial with virtual marketplace allowing DERs to buy and sell energy and flexibility on local and wholesale markets. DNO (acting as DSO) will manage constrained areas of the network using LMPs and by buying/selling flexible demand and generation form local marketplace.

TDI 2 [17] SE England, UK Pilot

Transmission Distribution Interface (TDI) 2 project aims to reduce congestion in an area of UK network with high penetration of DG. Project is to involve the DNO facilitating regional markets for active and reactive power taking on the role of DSO. 


\section{A. Ecogrid}

The Ecogrid trial project was carried out on the Danish island of Bornholm (which is fully integrated into the Nordic power system) for 1900 domestic and 100 industrial customers between 2011 and 2015 [18]. The aim of the project was to trial a regional real-time market where DERs including flexible demand customers are incentivised to react to variations in electricity prices based on local congestion. Trades are not carried out $\mathrm{P} 2 \mathrm{P}$ but through a central market with no local matching of supply/demand. However the aim of the real-time market is to provide local price signals to reduce congestion which does promote matching of local supply/demand by DERs. The prices are updated every 5 minutes (close to real-time) to reflect the need for regulating demand and supply based on system imbalances. It was aimed to alleviate congestion, allow DERs to be better utilised in the market and allow small scale DERs to be used that would otherwise have been left unused.

In the Ecogrid project, balancing is carried out by the TSO with the real-time market providing balancing at a higher resolution in parallel to the balancing (known as regulating in Denmark) market. This follows the 'Total TSO' model (described in Section II) where the TSO is optimising the dispatch of DERs. The advantage of this approach is that the real-time market and balancing markets can be coordinated centrally. The disadvantage is that for the trial to be carried out over the whole country, the complexity in computation and communications in managing the real-time markets could be excessive for a single TSO. In terms of congestion management it was found that real-time price signals reduced the overall peak load on Bornholm by $1.2 \%$ [3]. It was estimated that wind power curtailment using this scheme could be reduced by $80 \%$. However the project was not successful in reducing distribution feeder congestion.

\section{B. TDI 2 Project}

A recently funded UK project is the Transmission Distribution Interface (TDI 2 project) [17] run by UK Power Networks (UKPN) and National Grid. The aim of the project is to alleviate congestion in the UKPN operated south east of England network using active network management along with the increased role of the DNO to aggregate DERs. The project aims to create regional power markets managed by the DSO which allows procurement of reactive and active power services from DERs. The DSO then selects the optimum DER services to satisfy network constraints and presents the available services and costs at each GSP to the System Operator (SO), National Grid, who select the most economical option to satisfy grid constraints. This project along with the Cornwall local energy market [16] are at the cutting edge of demonstrating the potential of the role of the DSO in coordination distribution markets. The outcomes of these projects, both in very early stages at the time of writing, will have a large influence on the future of distributed electricity markets in the UK.
From the projects described above it is clear that distributed markets are developing around the world however the coordination of these markets to provide system wide benefits (constraint management, minimised grid reinforcement) continues to be an area for development. This paper will now go on to consider a tool that could be used by DSOs for coordinating future distributed markets, DLMPs.

\section{DistribUTION LMPS}

Locational marginal prices are used widely in transmission systems (e.g USA) to account for the spatial variation in costs of electricity primarily due to losses and constraints depending on distances and transmission capacities between loads and generation [19]. To date LMPs have not been used on distribution systems however they have potential in promoting DERs through price signals in local markets. This concept is under consideration in the UK in the Cornwall local energy market currently being developed by Centrica [16]. LMPs have the potential to reduce system losses (and increase efficiency) by reducing transport of electricity through directing investment in DERs. LMPs have the potential to reduce network upgrade requirements by promoting DERs in areas of constraint and to direct network investment by indicating areas where upgrades can be cost-effective.

LMPs can be defined as the marginal cost of supplying the next unit of demand at a certain location (node) taking into account supply bids and demand offers and the network characteristics including losses and limits [19]. The LMP has 3 components: the energy component, congestion component and loss component. The energy component is the load-weighted average of the system node prices and is the same at all locations. The congestion component reflects the marginal cost of congestion due to binding constraints (e.g line capacities, reserve requirements). The loss component is the marginal cost of any losses for a specific location. In this way a range of prices are possible that reflect the cost of providing electricity at each network location.

The theory underpinning LMP used in power markets was outlined in 1988 by Schweppe et al. [6]. More recently with the growth of DERs and the development of smart meters there has been work focused on introducing LMP to distribution networks. Work using DLMPs has been carried out on a basic 12-bus test distribution system [20], [21]. In Meng and Chowdhury [20] a modified optimal power flow (OPF) solver is used with social surplus, defined as the total benefits of the buyers minus the total costs of the sellers, set as the target function in an agent based DLMP calculation. In this work it was identified that congestion reduces social surplus due to the more expensive generators becoming marginal, furthermore increasing the load levels uniformly across the 12 bus system resulted in an increase in social surplus as the supplier surplus was increased. In Sahriatzadeh et al.[21] the Matpower OPF solver was again used on the same 12-bus system with demand responsive loads. In this work, adding responsive load gave a slight increase in social surplus due to 
loads at higher DLMP reducing consumption giving increased buyer surplus.

A more detailed analysis of DLMP has been carried out by Ntakou and Caramanis [22] on a 253-bus distribution feeder. The study considered participation of DERs in both real and reactive day ahead markets and showed that allowing distributed generators to provide VAr compensation significantly reduced system costs. The use of DLMPs for reducing congestion caused by electric vehicle $(\mathrm{EV})$ charging has been studied by Li et al. [23] where it is proposed that the market is managed by a DSO. In this study it was shown that DLMPs can alleviate congestion particularly for high penetration of EVs. In a study by Reno et al. [24] on DLMPs in optimal placement of residential PV systems it was seen that using DLMPs to inform the siting of PV significantly reduced line losses. In the aforementioned paper DLMPs were calculated on a distribution system within the NYISO (New York Independent System Operator) region and it was found that rewarding PV for both generation and loss reduction is beneficial to not only the PV operator but also the DSO.

Key variables in the implementation of DLMPs are the granularity of the regions, i.e. a primary or secondary feeder or an entire grid supply point (GSP) and the timesteps, i.e. every 5 minutes, 1 day or 1 year. These factors affect the level of information and communication systems required and complexity of market operation but also influence the effectiveness in responding to short term peak constraints. Work has been done by others in assessing the use of DLMPs as part of a large body of work on changes to regulations and pricing models for electricity markets [25]. The work is wide ranging and it is aimed to examine some of the findings using a simple network as part of ongoing research.

\section{A. Example network}

For the purposes of illustrating the possible effects of DLMPs an example 9-bus network (see Fig. 2) was modelled. The network is based on a $33 \mathrm{kV}$ section of the UK Generic Distribution System (UKGDS) EHV network [26] with the addition of 2 distributed generators.

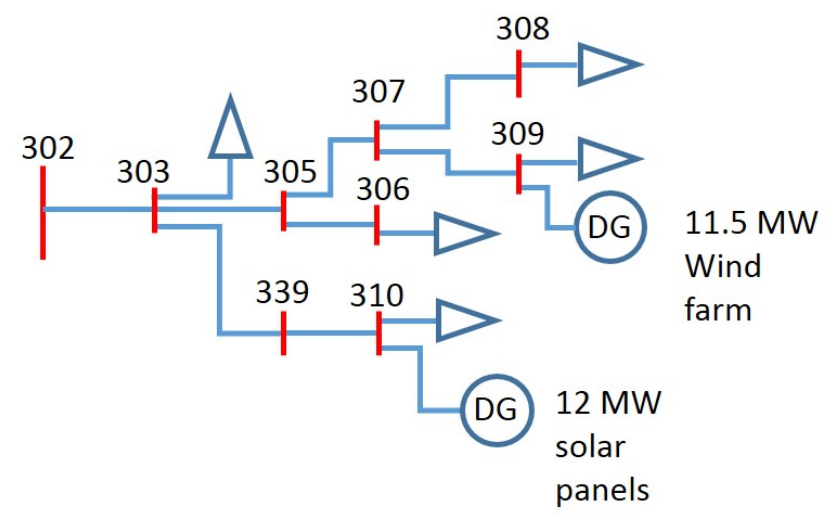

Fig. 2: Example 9-bus $33 \mathrm{kV}$ network
Each bus equates to a zone with separately calculated marginal price, therefore any load connected to bus 308 could in theory pay a different price users connected to bus 309 . Load profiles are aggregated for domestic and non-domestic profiles from the UK Energy Research Council data centre [27]. Generation profiles were created using the renewables output simulation tool developed by Staffell [28] and Pfenninger [29] for a location in the south west of Scotland.

\section{B. Case studies}

For the network in Fig. 2 a series of case studies were run over 24 hour periods with LMPs calculated at 30 minute intervals based on varying demand and generation profiles. The demand profiles are based on aggregating domestic and industrial loads. Case studies were run for a winters day with no DG, with DG, and for a test constrained case.

1) Case 1: Winters day - No DG: The network shown in Fig. 2 was modelled with the wind farm and solar panel DG removed. The resulting marginal prices at each bus are shown in Fig. 3 which shows that the loss component of the marginal prices is highest for bus 309 as the branch between 307 and 309 has the highest line impedance. The line impedances for branches 302-303, 305-306 and 307-308 are so low that the losses are negligible resulting in the same marginal costs for these bus pairings. In general marginal prices are higher with increasing distance from the import point at bus 302 .

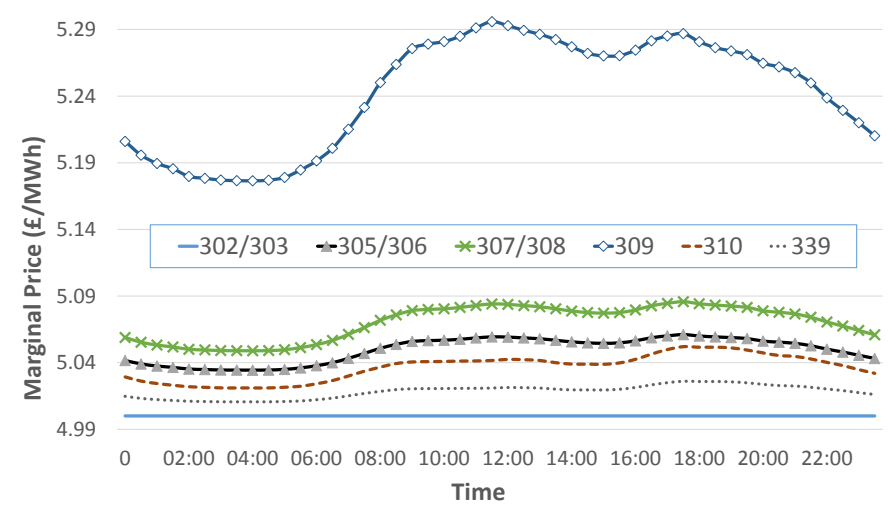

Fig. 3: Marginal prices on Jan 24th 2014 - no DGs

2) Case 2: Winters day - DG: The marginal prices at each bus in the network shown in Fig. 2 on a winters day with high wind output and minimal solar generation is shown in Fig. 4. The marginal cost at bus 309 is $10 \%$ lower than the import cost (cost at bus 302) due to loads at bus 309 avoiding import losses. A reverse of the situation in case 1 is seen as marginal prices rise with distance from bus 309 . The marginal prices at buses 339 and 310 are not affected by the low cost generation at bus 309 with the prices remaining the same as in case 1 .

3) Case 3: Winters day constrained: To observe the effect of a constrained network on marginal prices the solar panels 


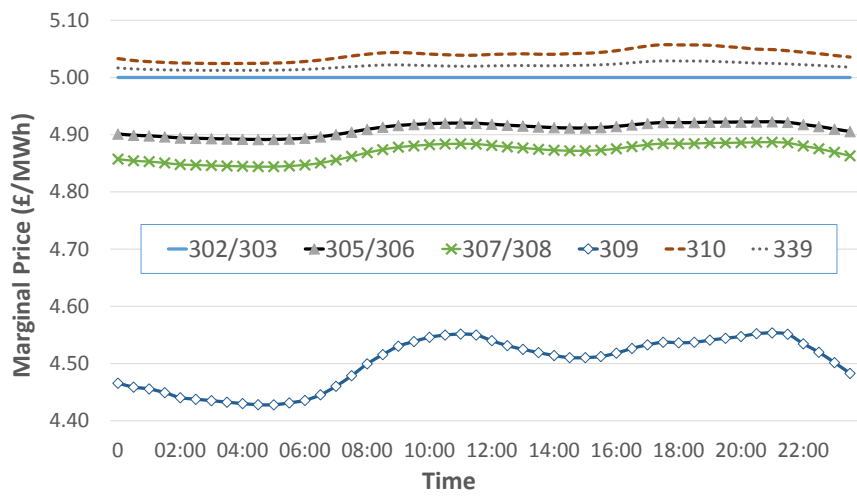

Fig. 4: Marginal prices on Jan 24th 2014 - with DGs

were moved to bus 308 and the output of the solar panels was increased to that of a sunny day in June. The results (Fig. 5) show that between 08:30 and 17:00 the marginal costs fall to zero for all buses downstream of bus 305 . At these times renewable generators with zero marginal costs (wind farm and solar panels) are being curtailed due to available generation exceeding the capacity of branch 303-305. Between 08:30 and 17:00, when solar output is highest, the cost of supplying an additional MWh is 0 for any load connected downstream of bus 305 as it will be served by an additional MWh of output from a renewable generator.

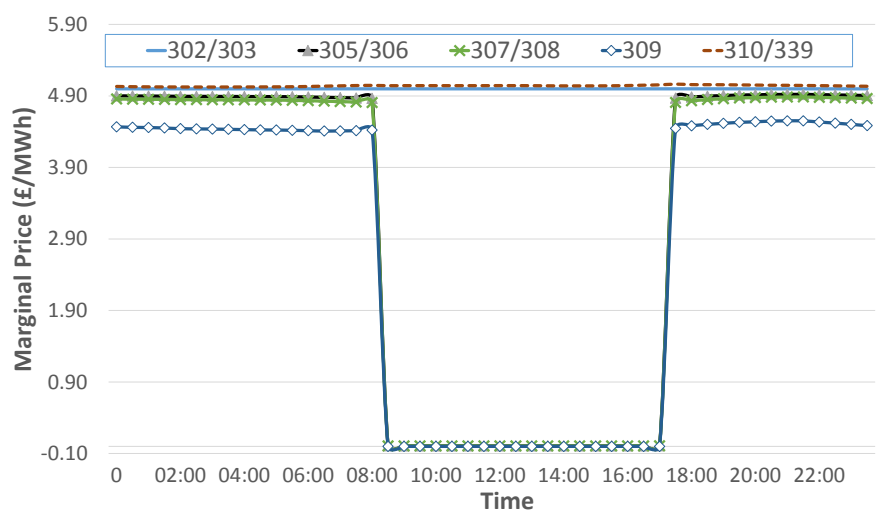

Fig. 5: Marginal prices Jan 24th 2014 with PV at bus 308

\section{Discussion}

The effect of losses and constraints on the marginal costs at different points on the 9-bus network considered have been illustrated using simple case studies. By installing a distributed generator at bus 309 the marginal price can be reduced by up to $14 \%$ (compared to the no DG case) due to reduced losses. However this is based on a day of the year with very high wind generation ( $90 \%$ of capacity) whereas based on a typical wind farm capacity factor of 30-40\% annual loss reduction will be closer to $5 \%$. An important option in the application of DLMPs is how these prices are passed on and the social implications. Bus 309 has the largest demand on the 9-bus test network equating to that of roughly 3000 homes. Should consumers be exposed to marginal prices they could be paying up to $£ 60$ a year more for energy than their counterparts at bus 303 or could benefit from a saving of $£ 100$ a year if they were fortunate enough to have a wind farm connected to the same bus.

In the constrained case (case 3) users could benefit from free electricity in areas where renewable generation is being curtailed. This is very likely to encourage any flexible demands (including storage) to consume electricity at these times which can provide network benefits such as peak smoothing if these flexible demands are shifted from peak times. One effect that could be observed with DLMPs is the prices returning to the import cost once the constraint is removed. The constraint could be removed either by increased demand in the constrained area (to match/exceed generation) or grid reinforcement. By incentivising the removal of constraints, DLMPs can provide system benefits by maximising output from renewables with associated reductions in carbon emissions. Removing constraints is a benefit for the local generators that are no longer being curtailed but not for the local demands that will no longer have free electricity. This is a risk to any investors in flexible demand or storage in a DMLP market as if enough local demand enters the market (or a network upgrade) to remove a constraint, the return on the investment for flexible demand customers in the constrained area will be reduced significantly.

If the consumers are on a fixed tariff it will be the supplier purchasing the energy that will benefit from any lower prices brought about by DLMPs. A fixed tariff may be preferable for households that can't afford the risk of being exposed to swings in energy prices. With competition in retail the choice should be available for those wishing to more actively participate in electricity markets such as with home PV, storage or flexible demand to have a tariff that is linked to marginal prices. This will encourage more investment in distributed generation and smoothing of peak loads by encouraging demand shifting based on response to price signals. For businesses (especially large electricity users) being exposed to marginal prices gives the opportunity to locate in areas with low marginal prices to reduce overheads. For electricity intensive industries, cheap electricity can be one of the main factors in siting a plant.

There is a trend in the UK of new domestic 'tracker' tariffs [30] which link to the wholesale price of electricity to give a more reflective charge to customers of actual energy costs. However basing tariffs solely on wholesale prices misses some of the major components of electricity costs namely distribution, capacity and ancillary service costs. By using DLMPs there is a closer reflection of distribution costs (including losses, constraints) although any tariff will need to include charges to cover other system costs. An issue with DLMPs may be with social acceptance as it will be those with access to capital that will benefit from exposure to DLMPs by having the opportunity to add flexibility (e.g. using batteries) or generation. Whereas those with limited access to capital will be pay higher prices if they are in 
areas with high DLMPs and no investment in DERs. In this case government regulation/intervention is essential from the beginning to ensure that no consumers are exposed to very high prices or investment support is available for DERs in those areas to bring prices down.

\section{CONCLUSIONS}

This paper has presented a review of distributed markets which is an area of growing interest at distribution level. The role of the DSO will be important in coordinating distributed markets and pricing of energy could be part of this transition. It is likely that a centralised approach with the DSO coordinating aggregated DERs will be favourable in the short term with a move towards distributed markets where DERs trade in forward markets and the DSO balances close to real-time as carried out by the TSO in the UK wholesale market.

The use of DLMPs has been seen to be a promising method of setting prices in distributed markets with the possibility of reducing system losses and alleviating constraints. However exposing consumers to these DLMPs poses some risks in terms of social acceptability and return on investment for flexible demand and storage. It should be ensured that system costs are spread fairly and a coordinated approach should be employed (e.g. via the DSO) to mitigate the investment risks for DERs.

\section{ACKNOWLEDGEMENT}

This work has been supported through the EPSRC Centre for Doctoral Training in Wind and Marine Energy Systems (EP/G037728/1).

\section{REFERENCES}

[1] UK Government, "Connecting new energy sources." [Online]. Available: http://www.publications.parliament.uk/pa/cm201617/cmselect/ cmenergy/267/26705.htm

[2] National Grid, "Future Energy Scenarios," Tech. Rep. July, 2015.

[3] Ecogrid EU, "EcoGrid EU : From Implementation to Demonstration," Tech. Rep., 2015. [Online]. Available: http://www.euecogrid.net/documents-and-downloads

[4] C. Zhang, Y. Ding, N. C. Nordentoft, P. Pinson, and J. Østergaard, "FLECH: A Danish market solution for DSO congestion management through DER flexibility services," Journal of Modern Power Systems and Clean Energy, vol. 2, no. 2, pp. 126-133, 2014.

[5] L. Kane and G. Ault, "A review and analysis of renewable energy curtailment schemes and Principles of Access: Transitioning towards business as usual," Energy Policy, vol. 72, pp. 67-77, 2014. [Online]. Available: http://dx.doi.org/10.1016/j.enpol.2014.04.010

[6] F. C. Schweppe, R. D. Caramanis, R. D. Tabors, and R. E. Bohn, Spot Pricing of Electricity, 1988.
[7] P. D. Martini, L. Kristov, and L. Schwartz, "Distribution systems in a high distributed energy resources future. Planning, Market Design, Operation and Oversight," Future Electric Utility Regulation, Tech. Rep. 2, 2015.

[8] Nord Pool, "Europes Leading Power Market." [Online]. Available: http://www.nordpoolspot.com/About-us/

[9] B. Sovacool, "Electricity market design for the prosumer era," Nature Publishing Group, no. March, pp. 1-6, 2016. [Online]. Available: http://dx.doi.org/10.1038/nenergy.2016.32

[10] Vandebron, "An online marketplace for energy; a world first in The Netherlands." [Online]. Available: http://vandebron.pr.co/72191an-online-marketplace-for-energy-a-world-first-in-the-netherlands

[11] Sonnen News, "AutoGrid and Sonnen Partner to Accelerate the Adoption of Energy Storage." [Online]. Available: https://www.sonnenbatterie.de/en-us/autogrid-and-sonnen-partneraccelerate-adoption-energy-storage

[12] LO3 Energy and Transactive Grid, "Brooklyn Microgrid - Transactive grid." [Online]. Available: http://brooklynmicrogrid.com/

[13] Open Utility, "A glimpse into the future of Britain's energy economy," Tech. Rep., 2016.

[14] Power Ledger, "Power + Blockchain." [Online]. Available: https://powerledger.io/

[15] PowerMatching City, "Hoogkerk." [Online]. Available: http://www.powermatchingcity.nl/site/pagina.php?id=41

[16] Centrica Distributed Energy \& Power, "Cornwall Local Energy Market." [Online]. Available: www.centrica.com/cornwall

[17] UK Power Networks (UKPN) and National Grid, "Transmission and Distribution Interface 2.0 (TDI) - Bid Document to Ofgem," RIIO Network Innovation Competition (NIC), Tech. Rep., 2016.

[18] P. Lund and O. S. Grand, "Deliverable D6.7 - Overall evaluation and conclusion," EcoGrid EU A Prototype for European Smart Grids., Tech. Rep., 2016.

[19] ISO New England Inc., "ISO New England Manual for Market Operations Manual M-11," Rev 47. October 6, 2013, Tech. Rep.

[20] F. Meng, S. Member, B. H. Chowdhury, and S. Member, "Distribution LMP-Based Economic Operation for future Smart Grid," 2011.

[21] F. Sahriatzadeh, P. Nirbhavane, and A. K. Srivastava, "Locational Marginal Price for Distribution System Considering Demand Response," pp. 1-5, 2012.

[22] E. Ntakou and M. Caramanis, "Price Discovery in Dynamic Power Markets with Low-Voltage Distribution-Network Participants," 2014.

[23] R. Li, Q. Wu, and S. S. Oren, "Distribution Locational Marginal Pricing for Optimal Electric Vehicle Charging Management," pp. 1-9, 2013.

[24] M. J. Reno, K. Coogan, J. Peppanen, and S. Grijalva, "Using Distribution LMP and Time-of-Delivery Pricing to Promote Optimal Placement and Increased Profitability of Residential PV Systems," 2014.

[25] Massachusetts Institute of Technology, "Utility of the future, An MIT Energy Initiative response to an industry in transition," Tech. Rep., 2016.

[26] Centre for Sustainable Electricity and Distributed Generation (SEDG), "UK Generic Distribution System (GDS) EHV network (ehv1). Unofficial Source." [Online]. Available: https://github.com/sedg/ukgds

[27] UKERC, "Energy Data Centre." [Online]. Available: http://ukerc.rl.ac.uk/DC/index.html

[28] I. Staffell and S. Pfenninger, "Using bias-corrected reanalysis to simulate current and future wind power output," Energy, vol. 114, pp. 1224-1239, 2016. [Online]. Available: http://dx.doi.org/10.1016/j.energy.2016.08.068

[29] S. Pfenninger and I. Staffell, "Long-term patterns of European PV output using 30 years of validated hourly reanalysis and satellite data," Energy, vol. 114, pp. 1251-1265, 2016. [Online]. Available: http://dx.doi.org/10.1016/j.energy.2016.08.060

[30] Simply Switch, "Octopus Energy Launches New Innovative Tracker Tariff." [Online]. Available: https://www.simplyswitch.com/octopusenergys-new-tracker-tariff-lets-customers-see-suppliers-profit-margins/ 